\title{
Disseminated histoplasmosis presenting as colonic
} pseudotumor

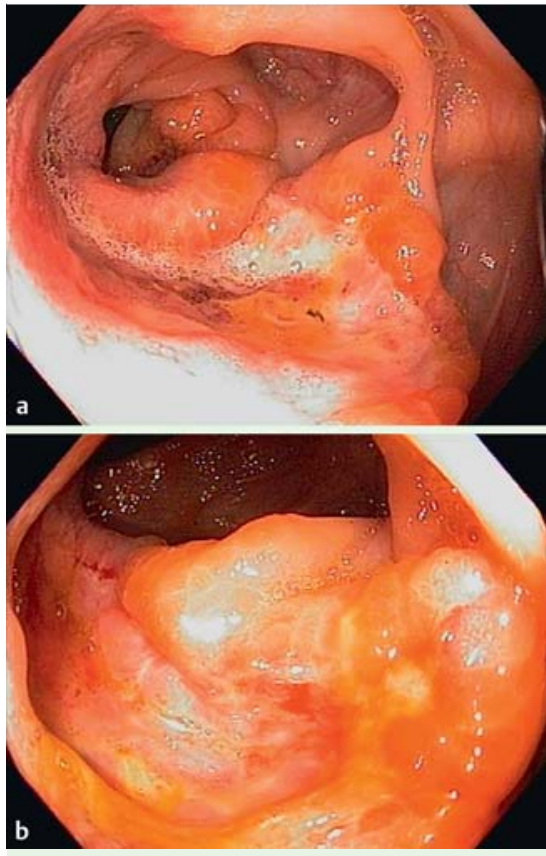

Fig. 1 Endoscopic appearances in a 56-yearold woman who presented with progressive cognitive decline and unintentional weight loss and was subsequently diagnosed as having acquired immune deficiency syndrome (AIDS) showing: a a large ulcerated area in the ascending colon; $\boldsymbol{b}$ the heaped-up margins of the ulcerated area.

Gastrointestinal histoplasmosis may be a rare initial manifestation in immunocompromised patients. We present a case of colonic histoplasmosis in a patient with human immunodeficiency virus (HIV), disseminated disease, and an unusual presentation.

A 56-year-old woman with no past medical history of note presented with progressive cognitive decline and an unintentional 95-pound weight loss over the previous year. The patient had been interacting less and was unable to walk because of a severely impaired gait. On physical examination, she was alert but disoriented with poor memory. She reported no gastrointestinal symptoms.

Her laboratory data were notable because they showed pancytopenia, but an initial neurologic and infectious work-up was negative. A magnetic resonance imaging (MRI) scan of her brain showed no acute intracranial pathology. Blood, urine, cere-

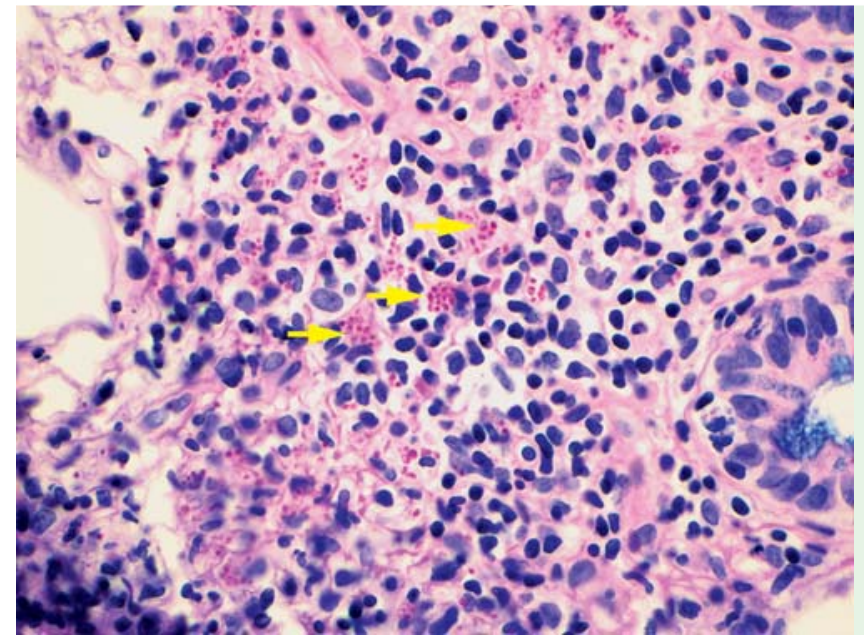

Fig. 2 Histologic appearance of a biopsy taken from the ulcerated area stained using the periodic acid-Schiff reaction (PAS) showing granulomatous inflammation with intracellular fungal organisms (arrowheads), consistent with Histoplasma capsulatum (magnification $\times 600$ ).

brospinal fluid (CSF), and sputum culture results were negative. A computed tomography (CT) scan showed diffuse bilateral ground-glass opacities in the lungs, splenomegaly, and thickening of the wall of the ascending colon. A bronchoscopy was performed, and bronchial washings and biopsies were negative for fungal organisms.

The patient underwent a colonoscopy, which demonstrated a large $10-\mathrm{cm}$ ulcerated area of mucosa with heaped-up edges in the ascending colon, suspicious for malignancy ( Fig. 1). Biopsies from this area showed prominent granulomatous reaction, but no dysplasia or malignancy was identified. Periodic acid-Schiff reaction (PAS), a fungal stain, revealed numerous intracytoplasmic and extracellular fungal organisms consistent with Histoplasma capsulatum ( $\bullet$ Fig. 2).

The patient was positive for HIV by enzyme-linked immunoassay (ELISA) and Western blot, with a CD4 count of 29, and was diagnosed with acquired immune deficiency syndrome (AIDS). She was treated initially with amphotericin B for disseminated histoplasmosis, which produced a clinical improvement, and was then changed to itraconazole as consolidation therapy.

Histoplasmosis is a common opportunistic infection in immunocompromised patients with HIV and has been an AIDSdefining illness. The causative organism $H$. capsulatum is the most common fungus in the USA and is endemic in the Mississippi and Ohio River Valleys. Disseminated histoplasmosis occurs from hematogenous spread of the inhaled fungal spores or reactivation of histoplasma granulomas. Gastrointestinal involvement in disseminated histoplasmosis is common (70\% - 90\% in autopsy studies); however, it is rarely identified during life [1]. The ileocecal area is commonly affected, presumably because of the abundant lymphoid tissue in this region. In our patient, diagnostic work-up did not reveal extracolonic histoplasmosis.

Gastrointestinal symptoms in disseminated histoplasmosis are nonspecific and may include abdominal pain, diarrhea, fever, weight loss, bleeding, and obstruction [2]. Endoscopic findings may include erythema, ulceration, stricture, or mass lesion, and may be misdiagnosed as colitis or malignancy [3]. Definitive diagnosis can be obtained by endoscopic biopsy of a lesion that subsequently demonstrates intracytoplasmic organisms. Disseminated histoplasmosis should be included in the differential diagnosis in immunocompromised patients with abnormal gastrointestinal imaging or symptoms. It is usually fatal if not diagnosed early and treated rapidly.

Endoscopy_UCTN_Code_CCL_1AD_2AZ

Competing interests: None 


\section{J. N. Winn, S. Akbar, S. Theivanayagam, R. G. Lim, B. R. Odum, M. L. Bechtold, A. Diaz-Arias, M. Arif}

Division of Gastroenterology, University of Missouri School of Medicine, Columbia, Missouri, USA

\section{References}

1 Kauffman CA. Histoplasmosis: a clinical and laboratory update. Clin Microbiol Rev 2007; 20: $115-132$

2 Assi M, McKinsey DS, Driks MR et al. Gastrointestinal histoplasmosis in the acquired immunodeficiency syndrome: Report of 18 cases and literature review. Diagn Microbiol Infect Dis 2006; 55: 195-201

3 Kahi CJ, Wheat LJ, Allen SD et al. Gastrointestinal histoplasmosis. Am J Gastroenterol 2005; 100: 220-231

\section{Bibliography}

DOI http://dx.doi.org/

10.1055/s-0032-1326495

Endoscopy 2013; 45: E155-E156

(c) Georg Thieme Verlag KG

Stuttgart · New York

ISSN 0013-726X

\section{Corresponding author}

\section{Arif, MD}

Division of Gastroenterology Hepatology CE405, DC 043.00

University of Missouri Health Sciences Center Five Hospital Drive

Columbia

Missouri 65212

USA

Fax: +1-573-884-4595

arifm@health.missouri.edu 